\title{
Optical/near-infrared observations of the black hole candidate XTE J1720-318: from high-soft to low-hard state ${ }^{\star}$
}

\author{
S. Chaty ${ }^{1}$ and N. Bessolaz ${ }^{2}$ \\ 1 AIM - Astrophysique Interactions Multi-échelles (UMR 7158 CEA/CNRS/Université Paris 7 Denis Diderot), CEA Saclay, \\ DSM/DAPNIA/Service d'Astrophysique, Bât. 709, L'Orme des Merisiers, 91191 Gif-sur-Yvette Cedex, France \\ e-mail: chaty@cea.fr \\ 2 Laboratoire d'Astrophysique, Observatoire de Grenoble, BP 53, 38041 Grenoble Cedex 9, France
}

Received 20 December 2005 / Accepted 6 April 2006

\section{ABSTRACT}

\begin{abstract}
Aims. To gain a better understanding of high-energy Galactic sources, we observed the Galactic X-ray binary and black hole candidate XTE J1720-318 in the optical and near-infrared, just after the onset of its X-ray outburst in January, 2003. These observations were obtained with the ESO/NTT as the Target of Opportunity, in February and April 2003.

Methods. We performed an accurate astrometry and analysed photometrical and spectroscopic observations. We then produced a colour-magnitude diagram, looked at the overall evolution of the multi-wavelength light curve, and analysed the spectral energy distribution.

Results. We discovered the optical counterpart in the $R$-band $(R \sim 21.5)$ and confirmed the near-infrared counterpart. We show that, for an absorption between 6 and $8 \mathrm{mag}$, XTE J1720-318 is likely to be an intermediate mass X-ray binary located at a distance between 3 and $10 \mathrm{kpc}$, hosting a main sequence star of spectral type between late B and early G. Our second set of observations took place simultaneously with the third secondary outburst present in X-ray and near-infrared light curves. The evolution of its spectral energy distribution shows that XTE J1720-318 entered a transition from a high-soft to a low-hard state in-between the two observations.
\end{abstract}

Key words. stars: binaries: close - stars: Hertzsprung-Russell (HR) and C-M diagrams - infrared: stars - X-rays: binaries X-rays: individuals: XTE J1720-318

\section{Introduction}

X-ray binaries are constituted of a compact object and a companion star, the former attracting matter from the latter, either through an accretion disc or the wind. They are usually divided in 2 sub-classes: high mass X-ray binaries and low mass $\mathrm{X}$-ray binaries, hosting early-type and late-type stars, respectively. Since accretion and ejection phenomena usually occur in these objects, they are ideal laboratories for studying relativistic phenomena and the formation and evolution of compact objects in binaries. However, to study them, we first have to derive the important parameters related to the nature of these systems, i.e. the distance, nature of the compact object, spectral type of the companion star, type of accretion, orbital parameters, etc. Because of the way they are formed, most of the observed Galactic X-ray binaries are located in the Galactic plane or even towards the Galactic centre, and therefore associated with very high absorption (up to $A_{\mathrm{v}} \sim 50 \mathrm{mag}$ ) because of the presence of gas and dust in this region. In this case, near-infrared (NIR) observations prove to be particularly useful, since the radiation is less absorbed at NIR wavelengths than at optical ones (see, e.g., Chaty et al. 2002). Furthermore, X-ray binaries have to be studied in a multi-wavelength context to disentangle all parts of the system emitting at various wavelengths: the accretion disc from high-energies to NIR, the companion star from ultra-violet to NIR, the jets from radio to X-rays, etc. (see, e.g., Chaty 2005).

* Based on ESO observations through programme \# 070.D-0340.
On January 9, 2003, the All Sky Monitor (ASM) of the Rossi-XTE satellite discovered a new source in the X-ray sky: XTE J1720-318, in the direction of the Galactic bulge (Remillard et al. 2003). The 2-12 keV flux was initially $\sim 130 \mathrm{mCrab}$ and reached $\sim 430 \mathrm{mCrab}$ on January 10, 2003. Spectroscopic observations with XMM-Newton were carried out on February 20, 2003, allowing Markwardt (2003) to estimate the column density of hydrogen on the line of sight: $N_{\mathrm{H}}=$ $1.33 \times 10^{22} \mathrm{~cm}^{-2}$. An iron line was detected at $6.2 \mathrm{keV}$ with $95 \mathrm{eV}$ equivalent width, and no low or high frequency oscillation was detected (Markwardt 2003). The $2-10 \mathrm{keV}$ flux was estimated to be $1.6 \times 10^{-9} \mathrm{erg} \mathrm{cm}^{-2} \mathrm{~s}^{-1}$ (Gonzalez-Riestra et al. 2003). The source was not detected during INTEGRAL/IBIS observations on February 28, 2003, but became visible at the end of the burst during IBIS surveys of the Galactic centre from the end of March 2003 (Goldoni et al. 2003). The source reached $\sim 25 \mathrm{mCrab}$ in the $15-40 \mathrm{keV}$ band and became detected in the 40-100 keV energy band at $\sim 30 \mathrm{mCrab}$ on April 6 and 7, 2003, indicating that the source had undergone a change of state, as suggested by Goldoni et al. (2003). The high-energy observations suggest that the compact object is a black hole, first because of its early spectral evolution that was very similar to black hole X-ray transients (Remillard et al. 2003), and also because of X-ray spectral parameters (Cadolle Bel et al. 2004) and the presence of an iron line (Markwardt 2003).

A radio counterpart was discovered with the VLA on January 15, 2003, and confirmed with ATCA on January 16, 2003: only one radio source included in the Rossi-XTE error box 
significantly varied from $0.32 \pm 0.04$ to $4.9 \pm 0.1 \mathrm{mJy}$ at $4.9 \mathrm{GHz}$ (Rupen et al. 2003). Radio observations took place from January to August 2003, allowing Brocksopp et al. (2005) to study the correlation between radio and X-ray fluxes.

A NIR counterpart was discovered then by Nagata et al. (2003) on January 18, 2003. Thirteen observations in $J, H$, and $K_{\mathrm{s}}$, until May 21, 2003, allowed them to measure the exponential decay following the burst, which was equal to 60 days.

In this paper, we will first describe our optical and NIR observations and data reduction in Sect. 2, then report on our astrometry, photometry, and spectroscopy results in Sect. 3. We will then focus on constraining the companion star spectral type in Sect. 4, and finally we will analyse the evolution of the XTE J1720-318 light curve and spectral energy distribution (SED) in Sect. 5.

\section{Observations and data reduction}

Our observations were carried out as part of the Target of Opportunity (ToO) programme 070.D-0340 (PI: S. Chaty) dedicated to the study of new Galactic high-energy sources and jet sources. They were triggered so as to be conducted at the same time as INTEGRAL ToO observations, and we asked for two periods of observations. The first set of observations took place on February 28, 2003, and the second one on April 24, 2003. On February 28, 2003, we obtained NIR photometry in $J-, H$-, and $K_{\mathrm{s}}$-bands with the spectra-imager SofI, and optical photometry in $B-, V-, R$-, and $I$-bands with EMMI, both installed on the NTT. On April 24, 2003, in addition to optical and NIR photometry, we also carried out NIR spectroscopy with SofI between 0.9 and $1.6 \mu \mathrm{m}$. We used the large field imaging of SofI's detector, giving an image scale of $0.288^{\prime \prime} \mathrm{pixel}^{-1}$ and a field of view of $4.94^{\prime} \times 4.94^{\prime}$, and the EMMI detector with an image scale of $0.32^{\prime \prime}$ pixel $^{-1}$ and a binning $2 \times 2$, giving a field of view of $9.9^{\prime} \times 9.1^{\prime}$.

Concerning the NIR observations, we repeated one set of observations for each filter with 9 different $30^{\prime \prime}$ offset positions, including XTE J1720-318, with an integration time of $90 \mathrm{~s}$ for each exposure, following the standard jitter procedure that allows us to cleanly subtract the blank NIR sky emission. We observed two photometrical standard stars of the faint NIR standard star catalogue of Persson et al. (1998): sj9157 on February 28, 2003, and sj9172 on April 24, 2003. We also performed rapid photometry in the $K_{\mathrm{s}}$-band for half an hour to detect rapid variations of magnitude by taking a set of 90 images with 2 s integration time each. We binned the images by three using a median filter, and we carried out aperture photometry.

Concerning the optical observations, we acquired $300 \mathrm{~s}$ exposures in each filter, except for the $B$-band (200 s), using a $2 \times 2$ binning to increase the sensitivity. We observed the standard star RU152 in $R$ - and $I$-bands. Since we did not have any standard star observations in the $B$ and $V$ filters, we used mean zero-points taken from the EMMI website ${ }^{1}$.

We used the Image Reduction and Analysis Facility (IRAF) suite to perform data reduction, carrying out standard procedures of optical and NIR image reduction, including flat-fielding and NIR sky subtraction. The zero-points we obtained are reported in Table 1. As we had only one standard star observation available for each night, we used characteristic extinction coefficients at la Silla: $\operatorname{ext}_{B}=0.214$, ext $_{V}=0.125$, ext $_{R}=0.091$, ext $_{I}=0.051$, $\operatorname{ext}_{J}=0.08, \operatorname{ext}_{H}=0.03$, and ext $\mathrm{es}=0.05$, to transform

\footnotetext{
1 www.ls.eso.org/lasilla/sciops/ntt/emmi/
}

Table 1. Zero-points derived from SofI and EMMI observations. $\mathrm{MJD}=\mathrm{JD}-2400000.5$.

\begin{tabular}{ccc}
\hline \hline & SofI observations & Emmi observations \\
\hline February 28, 2003 & $Z_{j}=2.229 \pm 0.009$ & \\
MJD 52 698 & $Z_{h}=2.259 \pm 0.007$ & \\
& $Z_{k_{s}}=2.862 \pm 0.006$ & \\
\hline April 24, 2003 & $Z_{j}=2.268 \pm 0.007$ & $Z_{b}=25.27 \pm 0.03$ \\
MJD 52 753 & $Z_{h}=2.451 \pm 0.009$ & $Z_{v}=25.98 \pm 0.01$ \\
& $Z_{k_{s}}=2.999 \pm 0.011$ & $Z_{r}=26.21 \pm 0.02$ \\
& & $Z_{i}=25.57 \pm 0.03$ \\
\hline
\end{tabular}

instrumental magnitudes into apparent magnitudes. The observations were performed through an airmass between 1 and 1.4.

Concerning the NIR spectroscopy, we took 12 spectra, half of them with an offset of $30^{\prime \prime}$ from the other half, to subtract the blank NIR sky, giving a total integration time of $180 \mathrm{~s}$. To extract spectra and perform wavelength and flux calibrations, we used the IRAF noao.twodspec package. We used the standard star sj9157 already mentioned above to perform flux calibration. Since the spectral type of this star is unknown, we used the calibrated $J, H$, and $K_{\mathrm{s}}$ magnitudes of Persson et al. (1998) to deduce its spectral type by using a colour-colour diagram taken from Cox (2000) and assuming a main sequence star with negligible interstellar absorption on the line of sight. We then synthesised a blackbody spectrum with effective temperature, corresponding to the determined spectral type. This flux calibration is quite crude, since NIR stellar spectra often present broad absorption features; however, it gives a good indication of the flux.

\section{Astrometry, photometry, and spectroscopy results}

We used the $K_{\mathrm{S}}$ image of the XTE J1720-318 field taken on January 21, 2003 (O'Brien et al. 2003) to identify XTE J1720-318 in our NTT images. We then determined the position of the XTE J1720-318 NIR counterpart by deriving the astrometric solution, using $\sim 12$ stars taken from the GSC2 catalogue: the position we measured was: $\alpha=17^{\mathrm{h}} 19^{\mathrm{m}} 58.988 \pm$ 0 s.008; $\delta=-31^{\circ} 45^{\prime} 01$.' $^{\prime} 21 \pm 0$.' 15 (equinox J2000). This position is consistent with other determinations (Table 2).

We discovered the optical counterpart in the $R$ - and $I$-bands at $\alpha=17^{\mathrm{h}} 19^{\mathrm{m}} 58.994 \pm 0.007, \delta=-31^{\circ} 45^{\prime} 01^{\mathrm{s}} .46 \pm 00^{\prime \prime} 15$ (equinox J2000), a position that is consistent with the NIR counterpart. We present $B V R I$ magnitudes in Table 3. The $R$ and $I$ magnitudes are consistent with detection limits given by Nagata et al. (2003): $R>18$ and $I>16.5$. We give a lower limit for the $B$ - and $V$-bands, as we did not detect any counterpart in these bands.

Since XTE J1720-318 is located close to the Galactic centre, we had to perform crowded field photometry to obtain precise NIR magnitudes, using the noao.daophot package. This procedure, described in Massey \& Davis (1992), consists of creating an empirical point-spread function with isolated bright stars, applying this model to the whole field, cancelling the contributions of neighbour stars, measuring the flux of the object itself, and then applying aperture correction (due to the use of a smaller aperture for measuring XTE J1720-318 magnitude than for standard stars). This procedure allows us to get photometry with better than $1 \%$ accuracy. We present the apparent $J, H$, and $K_{\mathrm{s}}$ magnitudes measured in February and April 2003 in Table 3. Uncertainties were determined from CCD readout and signal 
Table 2. Summary of astrometry results (equinox J2000) for the XTE J1720-318 NIR counterpart.

\begin{tabular}{cccc}
\hline \hline & This paper & Nagata et al. (2003) & O'Brien et al. (2003) \\
\hline$\alpha$ & $17^{\mathrm{h}} 19^{\mathrm{m}} 58^{\mathrm{s}} .988 \pm 0.008$ & $17^{\mathrm{h}} 19^{\mathrm{m}} 59^{\mathrm{s}} .000 \pm 0.014$ & $17^{\mathrm{h}} 19^{\mathrm{m}} 58^{\mathrm{s}} .994 \pm 0.004$ \\
$\delta$ & $-31^{\circ} 45^{\prime} 01^{\prime \prime} 21 \pm 0.0^{\prime} 15$ & $-31^{\circ} 45^{\prime} 01^{\prime \prime} 2 \pm 00^{\prime \prime} 2$ & $-31^{\circ} 45^{\prime} 01^{\prime \prime} 25 \pm 0.05$ \\
\hline
\end{tabular}

Table 3. Apparent $B, V, R, I, J, H$, and $K_{\mathrm{s}}$ magnitudes of XTE J1720-318 (MJD = JD - 2400 000.5).

\begin{tabular}{ccccccccc}
\hline \hline Date & MJD & $B$ & $V$ & $R$ & $I$ & $J$ & $H$ & $K_{\mathrm{s}}$ \\
\hline 20030228 & 52698 & & & & & $17.47 \pm 0.05$ & - & $16.00 \pm 0.06$ \\
20030424 & 52753 & $>23.2 \pm 0.4$ & $>23.1 \pm 0.4$ & $21.5 \pm 0.3$ & $20.6 \pm 0.1$ & $17.66 \pm 0.05$ & $16.99 \pm 0.07$ & $16.34 \pm 0.05$ \\
\hline
\end{tabular}

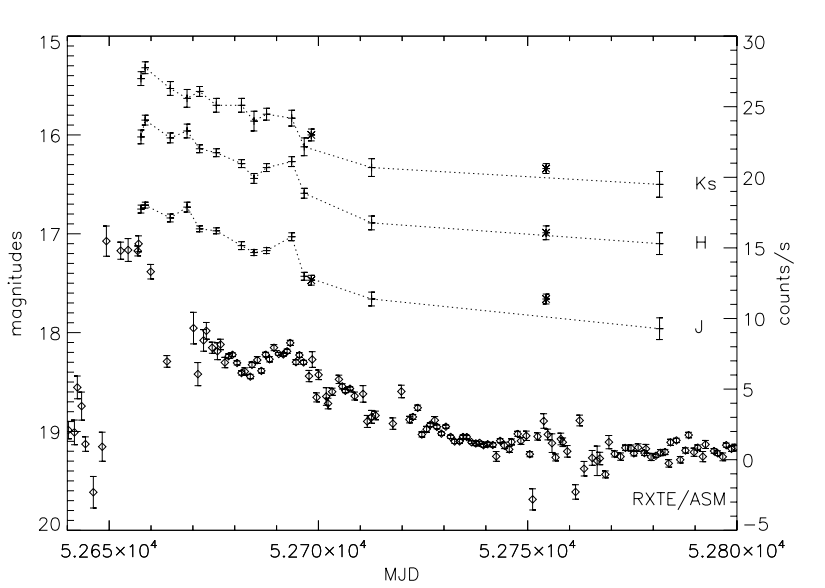

Fig. 1. Multi-wavelength XTE J1720-318 light curve. From bottom to top: Rossi-XTE X-ray light curve (indicated by diamonds); NIR $J, H$, and $K_{\mathrm{s}}$ light curves, respectively. NIR data taken from Nagata et al. (2003) are reported with "+", and data from this paper with "*". $\mathrm{MJD}=\mathrm{JD}-2400000.5$.

noise. We note that we do not include the $H$-band observation of February 28, 2003, here because of bad sky subtraction.

Our NIR photometrical observations are reported in Fig. 1, where we also included results from Nagata et al. (2003). Our magnitudes, indicated by " $*$ ", are consistent with measures from Nagata et al. (2003). We notice the similar behaviour of X-ray and NIR light curves, particularly during the first maximum when the coverage is more complete, and we point out that we observed an increase in NIR on April 24, 2003 (MJD 52 753), observed by chance at exactly the same time as an X-ray increase seen on the light curve.

To analyse the rapid $K_{\mathrm{s}}$ photometry, we performed aperture photometry of XTE J1720-318 and three other stars of different magnitudes in the field. We show the results in the left panel of Fig. 2: we see the same type of variability for XTE J1720-318 as for comparison stars, showing that this variability is more likely due to sky variations than to the X-ray binary itself. Dividing XTE J1720-318 flux by the means of comparison star fluxes, we corrected the NIR light curve of XTE J1720-318 from this sky variability, to detect XTE J1720-318 intrinsic variations, if any. The corrected light curve is presented in the right panel of Fig. 2. The mean instrumental $K_{\mathrm{s}}$ magnitude, obtained by median-filtering all images, is $18.93 \pm 0.04 \mathrm{mag}$, and the standard deviation of all measures is $\sim 0.11 \mathrm{mag}$. We can see a periodicity of $\sim 600 \mathrm{~s}$ in Fig. 2; however, since the interval of magnitude variation is around $0.3(3 \sigma)$, it is not significant enough to interpret it as XTE J1720-318 intrinsic variations. Furthermore, a discrete Fourier analysis of these data does not give significant evidence of periodicity. Absence of significant variability at the level of $+/-0.1$ mag is consistent with the similar analysis done earlier in the outburst by O'Brien et al. (2003).

Finally, concerning the spectroscopy, we show the flux and wavelength calibrated spectrum we obtained in Fig. 3. Although the signal-to-noise ratio was too small to detect any line, the spectrum allowed us to estimate an upper limit to XTE J1720-318 flux: $\sim 2 \times 10^{-16} \mathrm{erg} \mathrm{cm}^{-2} \mathrm{~s}^{-1} \AA^{-1}$.

\section{Constraints on the companion star spectral type}

We will now try to constrain the nature of the companion star, first with a colour-magnitude diagram, then by modelling the optical and NIR spectral energy distribution (SED).

\subsection{Colour-magnitude diagram}

XTE J1720-318 optical and NIR magnitudes allow us to constrain the nature of the binary system by comparing its absolute magnitudes with those of well-determined spectral type stars (see, e.g., Chaty et al. 2002). For this purpose, we use template absolute magnitudes related to spectral types (taken from Ruelas-Mayorga 1991 for the NIR and Cox 2000 for the optical, respectively). The conversion of apparent magnitudes $m$ to absolute magnitudes $M$ depends on both distance $d$ and interstellar absorption $A_{\mathrm{v}}$, via: $M=m+5-5 \log d(p c)-A_{\mathrm{v}}$. Concerning the interstellar absorption, we have three different estimates: First, XMM-Newton spectroscopy obtained in February 2003 gave $N_{\mathrm{H}}=1.24 \pm 0.02 \times 10^{22} \mathrm{~cm}^{-2}$ (Cadolle Bel et al. 2004). This column density corresponds to an absorption of $A_{\mathrm{v}}=6.9 \mathrm{mag}$ using the relation $A_{\mathrm{v}}=5.59 \times 10^{-22} N_{\mathrm{H}}$ (Predehl \& Schmitt 1995). Second, Nagata et al. (2003) obtained $A_{\mathrm{v}}=8$ by assuming a high temperature blackbody emission just after the X-ray burst. They also noted that extinction derived from the 2MASS survey is $A_{\mathrm{v}} \sim 6$.

Here, we will use only one observing epoch, on April 24, 2003, when the source is fainter, to minimise the accretion disc contribution in the observed NIR flux. Even if the object was still far from quiescence, in this way, we determine a lower limit for the companion star spectral type by assuming that the accretion disc emission reddens the NIR flux.

To derive the possible spectral types, we computed the absolute magnitudes of XTE J1720-318, taking various distances $d$ and absorption in the visible $A_{\mathrm{v}}$. The results are reported with " *" in the $\left(J-K_{\mathrm{s}}, K_{\mathrm{s}}\right)$ colour-magnitude diagram (CMD) presented in Fig. 4. The distance was computed between 1 and $10 \mathrm{kpc}$ (from bottom to top, respectively) and the absorption between 6 and 8 magnitudes (from right to left, respectively).

From this CMD, we first derive that, in any case, the companion star must belong to the main sequence. Furthermore, if the interstellar absorption is high, $A_{\mathrm{v}} \sim 8 \mathrm{mag}$, the spectral type 

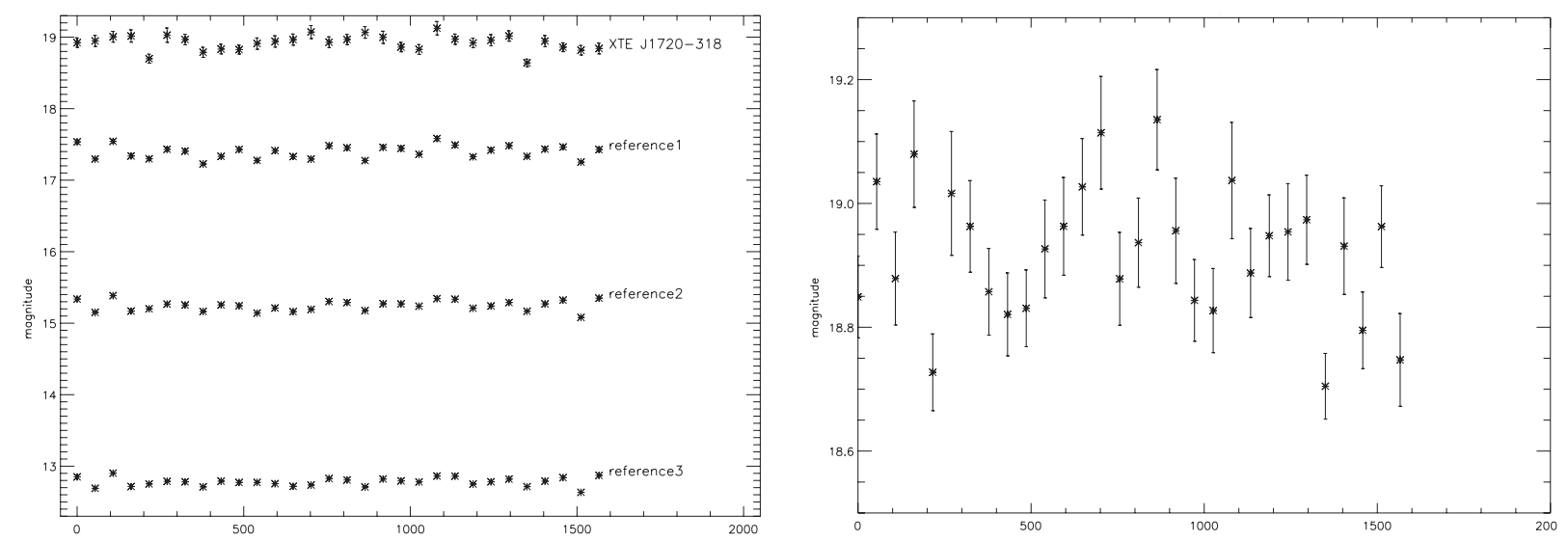

Fig. 2. Left panel: $K_{\mathrm{S}}$ light curve of XTE J1720-318 and three comparison stars of the field. Right panel: $K_{\mathrm{s}}$ light curve of XTE J1720-318 corrected from sky variations (the $y$-axis is in instrumental magnitude).

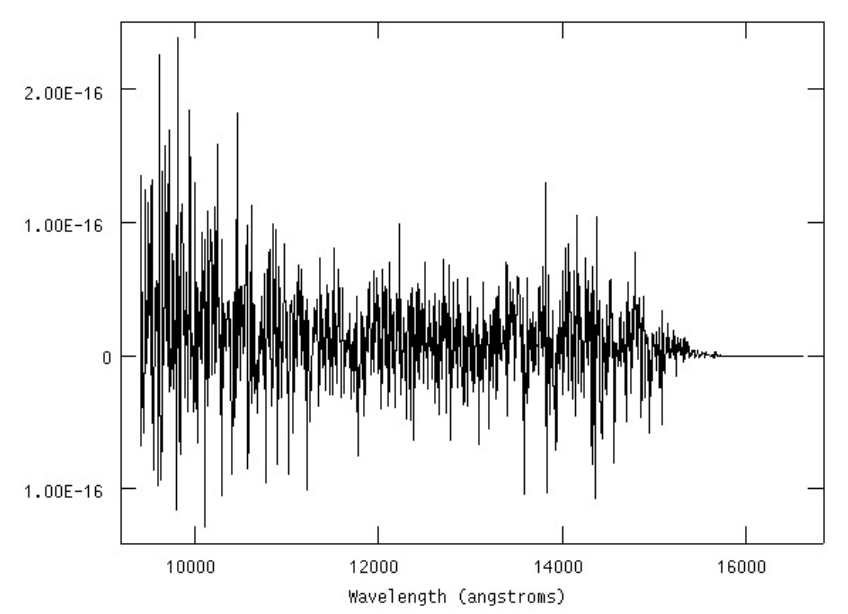

Fig. 3. NIR $K_{\mathrm{s}}$-band spectrum of XTE J1720-318. The flux is given in erg $\mathrm{cm}^{-2} \mathrm{~s}^{-1} \AA^{-1}$.

would be between late B and early A, and the source far away, between 6 and $10 \mathrm{kpc}$. With an intermediate value of the interstellar absorption, $A_{\mathrm{v}} \sim 7 \mathrm{mag}$, the spectral type would be between late $\mathrm{A}$ and early $\mathrm{F}$, and the distance between 5 and $7 \mathrm{kpc}$. Finally, with a small interstellar absorption, $A_{\mathrm{v}} \sim 6$ mag, the spectral type would be between late $F$ to early $G$, and the distance between 3 and $6 \mathrm{kpc}$. Therefore, we can conclude that, for an absorption between 6 and 8 mag, the XTE J1720-318 companion star is a main sequence star of spectral type between late B and early $\mathrm{G}$, located at a distance between 3 and $10 \mathrm{kpc}$. This estimate of distance makes the source closer than suggested by Nagata et al. (2003): it is therefore possible that the source is not located in the Galactic bulge. We point out that from this analysis, XTE J1720-318 can be added to the list of intermediate mass $\mathrm{X}$-ray binaries, like, e.g., V4641 Sgr (Chaty et al. 2003a).

\subsection{Temperature and interstellar absorption}

We now try to further constrain the absorption and spectral types by modelling XTE J1720-318's SED by an absorbed blackbody corrected from interstellar absorption. For each temperature, we calculate the associated blackbody spectrum that we multiply by the transmittance filter. Taking the interstellar absorption in the visible, we then derive absorption values for each wavelength, using Cardelli et al. (1989), and compute the magnitudes in each band.

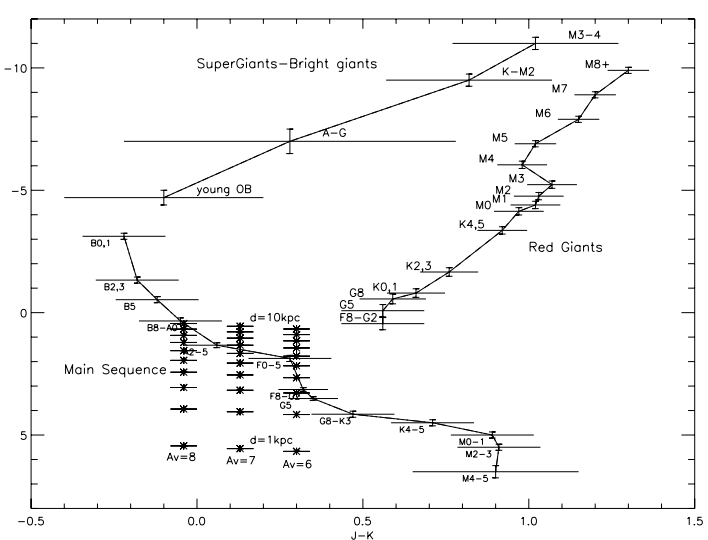

Fig. 4. Colour-magnitude diagram showing characteristic absolute magnitudes of various spectral types and XTE J1720-318 absolute magnitudes computed for interstellar absorption $A_{\mathrm{v}}$ between 6 and 8 mag ("*" from right to left) and distance between 1 and $10 \mathrm{kpc}$ ("** from bottom to top). We used the April 24, 2003, observations, when the object was fainter, to reduce the accretion disc contribution in the NIR flux. From this CMD we can conclude that XTE J1720-318 is an intermediate mass X-ray binary located at a distance between 3 and $10 \mathrm{kpc}$, and that the companion star is a main sequence star of spectral type between late B and early $\mathrm{G}$ (see text for more details).

We now want to compare these RIJHK magnitudes, computed for different effective temperatures and interstellar absorption, with apparent magnitudes. To do this, we calculate the discrepancy in each band by taking the $K_{\mathrm{s}}$ apparent magnitude and computing the quantity

$S=\left(R-R_{\mathrm{obs}}\right)^{2}+\left(I-I_{\mathrm{obs}}\right)^{2}+\left(J-J_{\mathrm{obs}}\right)^{2}+\left(H-H_{\mathrm{obs}}\right)^{2}$

for the $R-, I-, J-$, and $H$-bands, for all considered temperatures and absorptions. In Fig. 5, we show the various curves of $S$ versus effective temperatures $T$ (varying between 3300 and $9300 \mathrm{~K}$ ) parameterized by absorption $A_{\mathrm{v}}$ (varying between 5.5 and $8.5 \mathrm{mag}$ ).

We find that the best convergence, minimizing $\mathrm{S}$, is found towards the values of the parameters $\left(A_{\mathrm{V}} \sim 6, T \sim 4300 \mathrm{~K}\right)$, corresponding to an XTE J1720-318 companion star spectral type of late F - early $\mathrm{G} ;\left(A_{\mathrm{V}} \sim 7, T \sim 5600 \mathrm{~K}\right)$, corresponding to late $\mathrm{A}-$ early $\mathrm{F}$; and finally $\left(A_{\mathrm{V}} \sim 8, T \sim 8100 \mathrm{~K}\right)$, corresponding to late $\mathrm{B}$ and early $\mathrm{A}$. This is, therefore, consistent with the results given by the CMD. 


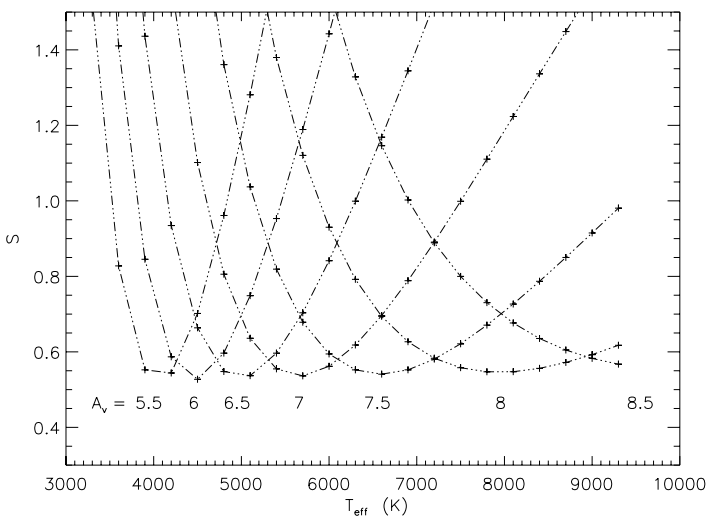

Fig. 5. Minimisation of the discrepancy between apparent and computed magnitudes, versus the blackbody effective temperature and interstellar absorption in the visible, given in magnitudes below each curve.

\section{Evolution of XTE J1720-318 SED}

In Fig. 6, we show the overall light curve of XTE J1720-318 from mid-January to the end of August 2003. First we can see that the light curve grossly has the form of a Fast-Rise Exponential-Decay light curve (so-called FRED), but superimposed on this FRED, the source exhibits a complex behaviour: after the main outburst on January 16 (MJD 52656 ), we can see both in X-rays and in NIR a secondary outburst on January 29 (MJD 52669 ), then a second one on February 22 (MJD 52 693), and finally a third one on April 24, 2003 (MJD 52 753), exactly at the time of our second epoch NIR observations. The main outburst and the last event are also associated with radio outbursts, and therefore with ejection events. We indicate the time of these events in Fig. 6 by O, 1, 2, and 3, respectively. There are other sources that exhibit clear secondary maxima in their X-ray light curves, such as A0620-00, GS 1124-68, GRO J0422+32 (Chen et al. 1997), 4U 1543-47 (Buxton \& Bailyn 2004), and XTE J1550-564 (Jain et al. 2001). However, XTE J1720-318 seems to be the second source after A0620-00 to exhibit clear multi-secondary maxima in the optical/NIR, correlated with the $\mathrm{X}$-rays, as seen in Fig. 6.

We also report the SED of XTE J1720-318 in Fig. 7 for the two observing epochs ("+" for February 28, 2003, and “*” for April 24, 2003, respectively), where we put together ESO/NTT optical/NIR observations from this paper, INTEGRAL/IBIS highenergy observations (Cadolle Bel et al. 2004), and ATCA/VLA radio data (Brocksopp et al. 2005). We will now describe the light curve and both SEDs and analyse XTE J1720-318's evolution between these two observing epochs.

\subsection{The February 2003 epoch: high-soft state}

Observations obtained with XMM-Newton, INTEGRAL/IBIS, and Rossi-XTE in February 2003 showed that the source spectrum was very soft (power law index of $\Gamma=2.7$ ): while the 2-12 keV flux was $\sim 100 \mathrm{mCrab}, I B I S$ detected a source at only $\sim 2.1 \mathrm{mCrab}$ in the $20-120 \mathrm{keV}$ band (Cadolle Bel et al. 2004). XTE J1720-318 was not detected in radio $(\leq 0.27 \mathrm{mJy}$ at $4.8 \mathrm{GHz}$, Brocksopp et al. 2005), which is consistent with typical high-soft state (see, e.g., Chaty et al. 2003b). This is corroborated by the analysis of XTE J1720-318 SED from February 28, to March 2, 2003 (reported with “+” in Fig. 7), where we notice the characteristic shape of the accretion disc emission in $\mathrm{X}$-rays (strong flux and soft spectrum), and the absence of radio emission.

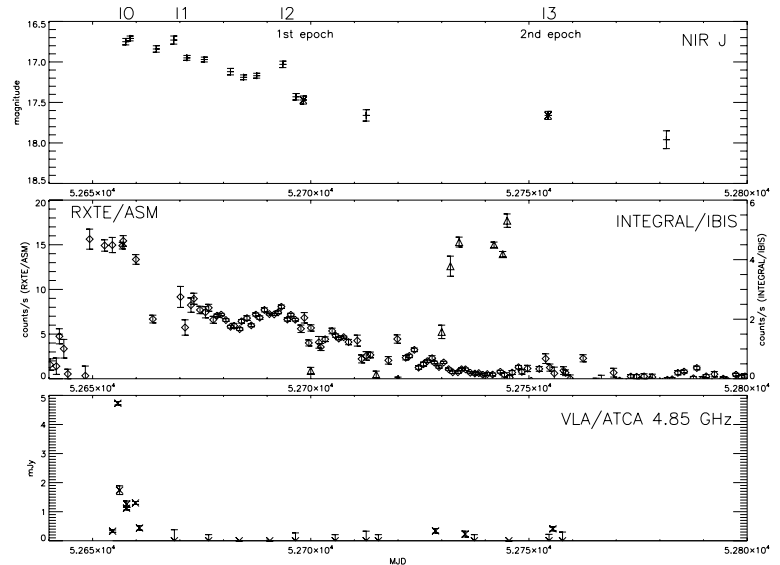

Fig. 6. Multi-wavelength light curve showing the outburst of XTE J1720-318 and its transition from high-soft to low-hard state. Top panel: NIR observations ("+": Nagata et al. 2003, "*”: this paper); Middle panel: high-energy observations (" $\diamond ": R X T E / A S M$, " $\triangle$ ": INTEGRAL/IBIS Cadolle Bel et al. 2004); Bottom panel: radio VLA/ATCA observations ("x": Brocksopp et al. 2005). We indicated at the top the times when the main outburst $(\mathrm{O})$ and the three secondary outbursts $(1,2,3$ respectively) occurred. MJD $=\mathrm{JD}-2400000.5$.

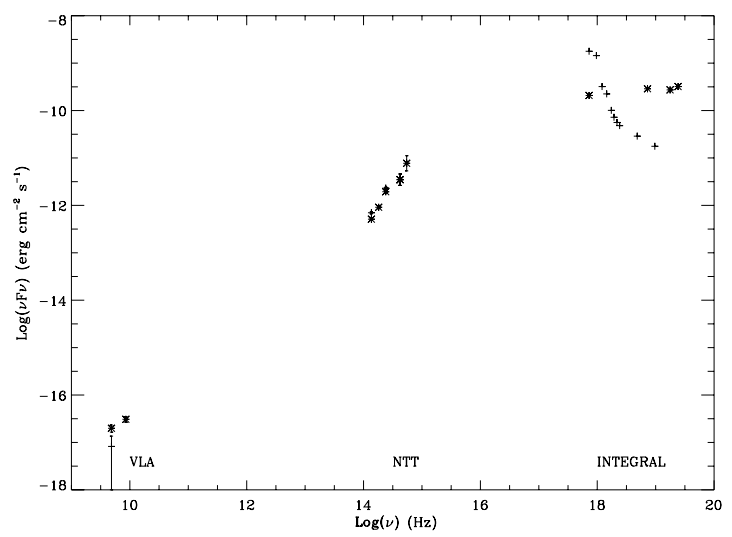

Fig. 7. Spectral Energy Distribution of XTE J1720-318 during the two observing epochs: the February 28, 2003, data are represented by "+", and the April 24, 2003, by “*”. The observations were taken nearly simultaneously with the VLA in the radio (Brocksopp et al. 2005), the NTT/EMMI and SOFI in the optical and NIR (this paper), and INTEGRAL/IBIS in the high-energy (Cadolle Bel et al. 2004). For the first epoch, the VLA data were taken on February 26, 2003, NTT/SOFI data on February 28, 2003, and INTEGRAL/IBIS data on February 28 - March 2, 2003. For the second epoch, VLA data were taken on April 26, 2003, NTT/SOFI data on April 24, 2003, NTT/EMMI on April 27, 2003, and INTEGRAL/IBIS data on February 28 - March 2, 2003. The February 28, 2003, observations correspond to the high-soft state: high and soft X-ray flux and no radio emission. On the contrary, the April 24, 2003, observations correspond to the low-hard state: low and hard X-ray flux and detection of radio emission. Optical and NIR fluxes were de-reddened, assuming an interstellar absorption in the visible of $A_{\mathrm{v}}=7 \mathrm{mag}$. It is remarkable that they remain the same in both states.

\subsection{The April 2003 epoch: low-hard state}

On the other hand, after March 25, 2003 (MJD 52723), XTE J1720-318 hard X-ray flux as seen by INTEGRAL/IBIS increased by a factor of 100 with respect to the high-soft state (Cadolle Bel et al. 2004), while the soft X-ray flux (RXTE/ASM) remained constant, as seen on the light curve of Fig. 6. Besides, we also observed a radio burst (Brocksopp et al. 2005), 
simultaneous with an X-ray burst and an increase in the NIR flux. Therefore, XTE J1720-318 seems to have entered a transition towards a low-hard state in-between these 2 observing epochs, as suggested by Goldoni et al. (2003). This is confirmed by the analysis of the SED (data of this second epoch are reported with "*" in Fig. 7): the source shows all the usual signs of the low-hard state. Firstly, we immediately notice that the source hardened in the high-energy domain with a high-energy power law index of $\Gamma=1.8$. Secondly, the radio emission is usually interpreted in this state as synchrotron emission emanating from a jet. From the SED, we can derive the power law index $\alpha$ (in $S_{v} \propto v^{\alpha}$ ): $\sim-0.3$ in the radio and $\sim 1.6$ in the optical/NIR. Therefore, the extrapolation of the radio flux towards the optical/NIR domain is significantly fainter than the observed optical/NIR flux. This strongly suggests that the synchrotron emission from the jet is contributing only for a small part, if any, in the NIR emission. It seems also likely that there is no contribution from the accretion disk in the optical/NIR domain, since the slope in the NIR and optical remarkably remains the same in both observing epochs, while the X-ray decreased at the same time. Therefore, the NIR emission is dominated by the contribution of the companion star, which is consistent with XTE J1720-318 being an intermediate mass X-ray binary.

To further understand this source, it would be useful i) to get spectroscopic observations of XTE J1720-318 in quiescence to better characterise the companion star when the photospheric flux of the star dominates and ii) to observe the radial velocity of the binary system to derive the mass function and orbital parameters. With these parameters we will be able to further analyse its SED (as in, e.g., Chaty et al. 2003b, with the source XTE J1118+480).

\section{Conclusions}

We have reported optical and NIR observations of the X-ray binary XTE J1720-318, taken as Target of Opportunity observations following the January 2003 outburst of this source. By performing accurate astrometry, we discovered the optical counterpart in the $R$-band $(R \sim 21.5)$ and confirmed the nearinfrared counterpart. From photometric observations, analysis of a colour-magnitude diagram, and a basic modelling of its SED, we found that, for an absorption between 6 and 8 mag, XTE J1720-318 is likely to be an intermediate mass X-ray binary, hosting a black hole and a main sequence star of spectral type between late $\mathrm{B}$ and early $\mathrm{G}$, located at a distance between 3 and $10 \mathrm{kpc}$. We also analysed the XTE J1720-318 X-ray and near-infrared light curves: this source exhibited three secondary outbursts, and our second set of observations took place simultaneously with the third one. Comparing the SEDs during and after its outburst, we confirm the change of state of this source, from high-soft to low-hard state.

Acknowledgements. S.C. thanks the ESO staff and especially Malvina Billères, Cédric Foellmi, Lisa Germany, Olivier Hainaut, Gaspare Lo-Curto, and Emanuela Pompei for performing the ToO observations. We are grateful to Marion Cadolle-Bel and Stéphane Corbel for making IBIS and ATCA data, respectively, available to us prior to publication. IRAF is distributed by the National Optical Astronomy Observatories, which are operated by the Association of Universities for Research in Astronomy, Inc., under a cooperative agreement with the National Science Foundation. This research has made use of NASA's Astrophysics Data System Bibliographic Services. XTE Results were provided by the ASM/RXTE teams at MIT and at the RXTE SOF and GOF at NASA's GSFC.

\section{References}

Brocksopp, C., Corbel, S., Fender, R., et al. 2005, MNRAS, 356, 125 Buxton, M. M., \& Bailyn, C. D. 2004, ApJ, 615, 880

Cadolle Bel, M., Rodriguez, J., Sizun, P., et al. 2004, A\&A, 426, 659

Cardelli, J. A., Clayton, G. C., \& Mathis, J. S. 1989, ApJ, 345, 245

Chaty, S. 2005, in 2005 Very High Energy Phenomena in the Universe; Proceedings of the XLth Rencontres de Moriond

Chaty, S., Mirabel, I. F., Goldoni, P., et al. 2002, MNRAS, 331, 1065

Chaty, S., Charles, P. A., Martí, J., et al. 2003a, MNRAS, 343, 169

Chaty, S., Haswell, C. A., Malzac, J., et al. 2003b, MNRAS, 346, 689

Chen, W., Shrader, C. R., \& Livio, M. 1997, ApJ, 491, 312

Cox, A. N. 2000, Allen's astrophysical quantities (Allen's astrophysical quantities, 4th ed., ed. A. N. Cox (New York: AIP Press; Springer)

Goldoni, P., Goldwurm, A., Kuulkers, E., et al. 2003, The Astronomer's Telegram, 153

Gonzalez-Riestra, R., Rodriguez-Pascual, P. M., Santos-Lleo, M., et al. 2003, IAU Circ., 8080, 1

Jain, R. K., Bailyn, C. D., Orosz, J. A., McClintock, J. E., \& Remillard, R. A. 2001, ApJ, 554, L181

Markwardt, C. B. 2003, The Astronomer's Telegram, 115, 1

Massey, P., \& Davis, L. 1992, A User's Guide to Stellar CCD Photometry with IRAF

Nagata, T., Kato, D., Baba, D., et al. 2003, PASJ, 55, L73

O'Brien, K., Clarke, F., Fender, R., et al. 2003, The Astronomer's Telegram, 117 Persson, S. E., Murphy, D. C., Krzeminski, W., Roth, M., \& Rieke, M. J. 1998, AJ, 116, 2475

Predehl, P., \& Schmitt, J. 1995, A\&A, 293, 889

Remillard, R. A., Levine, A. M., Morgan, E. H., Smith, E., \& Swank, J. 2003, IAU Circ., 8050

Ruelas-Mayorga, R. A. 1991, Rev. Mex. Astron. Astrofis., 22, 27

Rupen, M. P., Brocksopp, C., Mioduszewski, A. J., et al. 2003, IAU Circ., 8054 